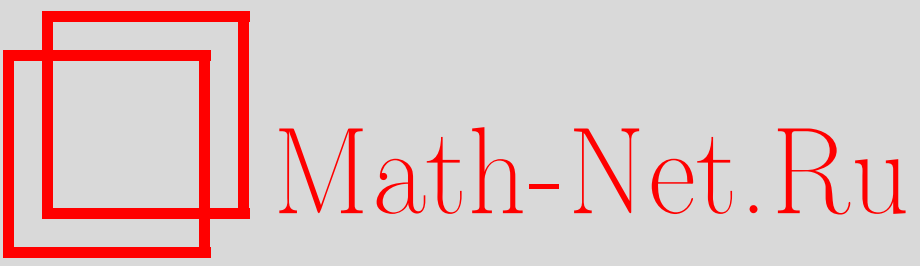

С. А. Богатый, Метрически однородные пространства, УМН, 2002, том 57, выпуск 2, 3-22

DOI: https://doi.org/10.4213/rm495

Использование Общероссийского математического портала Math-Net.Ru подразумевает, что вы прочитали и согласны с пользовательским соглашением

http://www.mathnet.ru/rus/agreement

Параметры загрузки:

IP : 35.173 .219 .149

26 апреля 2023 г., 14:04:15 


\title{
МЕТРИЧЕСКИ ОДНОРОДНЫЕ ПРОСТРАНСТВА
}

\section{С. А. БОГАТЫЙ}

\begin{abstract}
Обсуждается задача описания свойств класса метрических пространств, в рамках которого можно аксиоматически провести конструкцию Урысона построения универсального однородного метрического пространства (в данном классе). Одним из основных таких свойств является возможность склейки двух метрик, заданных на замкнутых подмножествах и совпадающих на пересечении.

Обсуждается проблема единственности (счетного, полного) однородного пространства, универсального в заданном классе метрических пространств. Рассмотрена задача продолжения сдвига Клиффорда компактного подмножества (ултраметрического) пространства Урысона до сдвига Клифффорда всего пространства Урысона.

Библиография: 66 названий.
\end{abstract}

На рубеже XIX и XX столетий основной задачей геометрии была задача ее аксиоматического построения. Такое построение было предложено почти одновременно несколькими учеными из разных стран, хотя большинство математиков связьвает его с книгой Давида Гильберта [1], которая ближе всех соответствует "Началам" Евклида. Возможно, что именно подход Евклида, избегавшего "процессы" и предпочитавшего рассмотрение "состояний”, обусловил отказ Гильберта от понятия "движения" в построении основ геометрии. Однако еше со времени Амадео Пеано была осознана важность понятия "движение”. Так, в схеме Пиери (ученика Пеано) основными понятиями являются "точка" и "движение"; в схеме В.Ф. Кагана - "точка", "расстояние” и "движение".

Задача выделения евклидовой, эллиптической и гиперболической групп среди групп изометрий всех римановых многообразий восходит к Риману и Гельмгольцу. В своей знаменитой работе С. Ли решил эту задачу (называемую теперь задачей Гельмгольца-Ли [2]) и показал, что всякое риманово многообразие со свойством локальной свободной подвижности является локально евклидовым, сферическим или гиперболическим.

В 1930 г. А.Н. Колмогоров предложил тополого-алгебраическую схему построения геометрии [3], в которой изометрии играют основную роль. В начале 40-х годов Г. Буземанн и Г. Биркгоф предложили построение (евклидовой, гиперболической и сферической) геометрии на основе понятия метрики и движения [4]-[7], [8; с. 72, 95-97]. Так,

Работа выполнена при поддержке Российского фонда фундаментальных исследований (грант № 00-01-00289). 
Биркгоф показал, что $n$-мерные евклидовы, сферические и гиперболические геометрии характеризуются как метрические пространства, в которых любые две точки соединяются (локально единственным) метрическим отрезком и всякая изометрия подмножеств продолжается до изометрии (на) всего пространства. Как отмечает Биркгоф, эта гипотеза свободной подвижности подобно Прометею появляется в разных видах в доказательстве различных теорем. В системах Колмогорова и Буземана предполагается меньшая подвижность (у Буземана требуется лишш возможность продолжения изометрии двух- и трехточечного подмножества), но предполагается локальная компактность пространства. Ванг [9]-[10] и Титс [11]-[13] доказали глубокие теоремы о геометрии связных локально компактных метрических пространств, в которых всякая изометрия, заданная на двух точках, продолжается до изометрии всего пространства. Всякое такое связное локально компактное пространство является симметрическим римановым многообразием [14]-[21], [22; §8.12], [23; 18$]$.

На основе понятия симметрии (не только центральной, но и осевой) строится геометрия в школе немецкого геометра Фридриха Бахмана [24].

Особняком стоит работа П. С. Урысона [25], в которой построено полное универсальное метрически однородное пространство $U$ и доказана его единственность. Как отмечает А. М. Вершик [26; с. 57], “эта работа не получила широкой известности, хотя того заслуживает". Ясно, что для пространства Урысона $U$ должны нарушаться некоторые аксиомы (свойства) в схемах Колмогорова (нет локальной компактности), Биркгофа (нет локальной единственности отрезков и метрической однородности относительно всех подмножеств), Ванга и Титса (нет локальной компактности). Однако метрическая однородность пространства Урысона относительно класса всех конечных подмножеств и единственность полного универсального пространства с этим свойством позволяют считать, что пространство Урысона есть один из естественных претендентов на реализацию бесконечномерной геометрии. Все сказанное выше показывает естественность и важность описания (классификации) всех (полных) метрически однородных пространств. В [27] по схеме Урысона было построено универсальное ультраметрическое пространство $U_{0}$. Мы показьваем, что схема построений и доказательств Урысона в значительной степени может быть формализована, однако полученное описание не позволяет предъявить в явном виде все метрически однородные пространства. Поэтому задача описания всех метрически однородных пространств не может считаться решенной. Под метрической однородностью мы понимаем здесь однородность относительно всех конечных подмножеств. Мы показьваем, что в пространстве Урысона $U$ (в пространстве $U_{0}$ ) имеется такое счетное замкнутое дискретное подмножество, не все изометрии с которого продолжаются на все пространство $U$ (на все пространство $U_{0}$ ). Это дает ответ на вопрос из [28] и усиливает полученное там решение задачи Урысона [28], [29]: изометрия счетного замкнутого подмножества пространства Урысона не всегда имеет продолжение.

Знаменитая теорема Нёбелинга-Понтрягина-Лефшеца-Менгера дает топологическое описание подмножеств евклидовых пространств. Теорема Менгера метрически характеризует подмножества евклидова (гильбертова) пространства $\mathbb{R}^{n}\left(\ell_{2}\right)$. Известная теорема Смирнова [30] равномерно характеризует подмножества евклидова (гильбертова) пространства. В связи с этим было бы интересно решить задачу описания всех равномерно однородных пространств. 
1. Единственность. В этой части даются критерии изометричности однородных метрик и существования изометрического вложения. Все пространства по умолчанию предполагаются метрическими сепарабельными.

Для метрического пространства $\left(Y, \varrho_{Y}\right)$ пусть

$$
\mathscr{A}_{Y}=\left\{\left(K,\left.\varrho\right|_{K}\right): K \subset Y-\text { конечное подмножество } Y\right\}
$$

Будем говорить, что метрическое пространство $\left(Y, \varrho_{Y}\right)$ является метрически (инбективно) однородным по отношению $\kappa$ классу подмножеств $\mathscr{K}$, если для всякой изометрии $f: K \hookrightarrow Y$ всякого подмножества $K \subset Y$ из класса $\mathscr{K}$ существует такая изометрия (в) $F: Y \rightarrow Y$, что $f=\left.F\right|_{K}$. Если $\mathscr{K}=\mathscr{A}_{Y}$, то будем говорить просто о метрической (инвективной) однородности.

Скажем, что метрическое пространство $Y$ является (слабым) абсолютным метрическим әкстензором для класса метрических пространств $\mathscr{K}(Y \in(\mathrm{W}) \mathrm{AME}(\mathscr{K}))$, если для всякого пространства $X \in \mathscr{K}$ и всякой изометрии $f: A \rightarrow Y$, заданной на (замкнутом) подмножестве $A \subseteq X$, сушествует такая изометрия $F: X \rightarrow Y$, что $\left.F\right|_{A}=f$.

Легко видеть, что всякое метрически инбективно однородное пространство $Y$ является $\mathrm{AME}\left(\mathscr{A}_{Y}\right)$-пространством. Для счетных и полных пространств верно и обратное.

Лемма 1.1. Пусть $\left(X, \varrho_{X}\right)$ - счетное метрическое пространство, а $\left(Y, \varrho_{Y}\right)$ - такое метрическое пространство, что $Y \in \operatorname{AME}\left(\mathscr{A}_{X}\right)$. Тогда для всякой изометрии $f: K \rightarrow Y$ конечного подмножсества $X$ существует такая изометрия (в) $F: X \rightarrow Y$, чтоо $f=\left.F\right|_{K}$.

ДокАЗАтельство. Занумеруем точки $X \backslash K=\left\{x_{1}, \ldots, x_{n}, \ldots\right\}$. Теперь по индукции построим такую последовательность изометрических вложений $f_{n}: K_{n} \rightarrow Y$, что $\left.f_{n}\right|_{K_{n-1}}=f_{n-1}$, где $K_{n}=K \bigcup_{i=1}^{n}\left\{x_{i}\right\}$ и $K_{0}=K, f_{0}=f$.

Пусть отображение $f_{n}$ уже построено. Из включений $K_{n+1} \in \mathscr{A}_{X}$ и $Y \in \operatorname{AME}\left(\mathscr{A}_{X}\right)$ вытекает существование такой изометрии $f_{n+1}: K_{n+1} \rightarrow Y$, что $f_{n+1}(k)=f_{n}(k)$ для всякой точки $k \in K_{n} \subset K_{n+1}$. Совокупность изометрических вложений $\left\{f_{n}\right\}_{n=1}^{\infty}$ корректно определяет такое изометрическое вложение $F: X \rightarrow Y$, что $\left.F\right|_{K}=f$.

Лемма 1.2. Пусть $\left(X, \varrho_{X}\right)$ - метрическое пространство, $C \subset X$ - такое замкнутое вполне ограниченное подмножество, что $|X \backslash C| \leqslant \aleph_{0}, a\left(Y, \varrho_{Y}\right)-$ такое ультраметрическое пространство, что $Y \in \operatorname{AME}\left(\mathscr{A}_{X}\right)$. Тогда для всякой изометрии $f: C \rightarrow Y$ существует такая изометрия (в) $F: X \rightarrow Y$, что $f=\left.F\right|_{C}$.

ДокаЗАТЕльство. Из включения $Y \in \operatorname{AME}\left(\mathscr{A}_{X}\right)$ вытекает $\mathscr{A}_{X} \subseteq \mathscr{A}_{Y}$. Так как метрическое пространство является ультраметрическим тогда и только тогда, когда ультраметрическим является всякое его трехточечное подмножество, то пространство $X$ также является ультраметрическим. Занумеруем точки $X \backslash C=\left\{x_{1}, \ldots, x_{n}, \ldots\right\}$. Теперь по индукции построим такую последовательность изометрических вложений $f_{n}: C_{n} \rightarrow Y$, что $\left.f_{n}\right|_{C_{n-1}}=f_{n-1}$, где $C_{n}=C \bigcup_{i=1}^{n}\left\{x_{i}\right\}$ и $C_{0}=C, f_{0}=f$.

Пусть отображение $f_{n}$ уже построено. Обозначим $\varepsilon_{n}=\varrho\left(x_{n+1}, C_{n}\right)>0$ и возьмем в $C_{n}$ конечную $\varepsilon_{n}$-сеть $B_{n}$. Из включений $B_{n} \cup x_{n+1} \in \mathscr{A}_{X}$ и $Y \in \operatorname{AME}\left(\mathscr{A}_{X}\right)$ вытекает сушествование такой изометрии $g_{n}: B_{n} \cup x_{n+1} \rightarrow Y$, что $g_{n}(b)=f_{n}(b)$ 
для всякой точки $b \in B_{n} \subseteq C_{n}$. Отображения $f_{n}$ и $g_{n}$ корректно определяют отображение $f_{n+1}: C_{n+1} \rightarrow Y$. Покажем, что это отображение является изометрией. Для точки $c \in C_{n}$ возьмем такую точку $b \in B_{n}$, что $\varrho(c, b)<\varepsilon_{n}$. Из последнего неравенства и равнобедренности треугольников в ультраметрическом пространстве $X$ вытекает равенство $\varrho\left(c, x_{n+1}\right)=\varrho\left(b, x_{n+1}\right)$. Согласно свойствам отображений $f_{n}$ и $g_{n}, \varrho\left(f_{n}(c), f_{n}(b)\right)=\varrho(c, b)<\varepsilon_{n}$ и $\varrho\left(g_{n}(b), g_{n}\left(x_{n+1}\right)\right)=\varrho\left(b, x_{n+1}\right) \geqslant \varepsilon_{n}$. Из равнобедренности треугольников в ультраметрическом пространстве $Y$ вытекает равенство $\varrho\left(f_{n+1}(c), f_{n+1}\left(x_{n+1}\right)\right)=\varrho\left(g_{n}(b), g_{n}\left(x_{n+1}\right)\right)=\varrho\left(b, x_{n+1}\right)=\varrho\left(c, x_{n+1}\right)$. Совокупность изометрических вложений $\left\{f_{n}\right\}_{n=1}^{\infty}$ корректно определяет такое изометрическое вложение $F: X \rightarrow Y$, что $\left.F\right|_{C}=f$.

Лемма 1.3. Пусть $\left(X, \varrho_{X}\right)$ - метрическое пространство, а $\left(Y, \varrho_{Y}\right)-$ такое полное метрическое пространство, что $Y \in \operatorname{AME}\left(\mathscr{A}_{X}\right)$. Тогда для всякой изометрии $f: K \rightarrow Y$ конечного подмножсества $X$ существует такая изометрия (в) $F: X \rightarrow Y$, чтоо $f=\left.F\right|_{K}$.

ДокАЗАТЕльство. Выберем в $X \backslash K$ счетное всюду плотное подмножество $S$. Согласно лемме 1.1 сушествует такая изометрия $g: K \cup S \rightarrow Y$, что $\left.g\right|_{K}=f$. Из полноты пространства $Y$ и равномерной непрерьвности отображения $g$ вытекает существование продолжения $F: X \rightarrow Y$, которое оказывается искомой изометрией.

СлЕДСТВИЕ 1.4. Пусть $\left(Y, \varrho_{Y}\right)$ - метрически (инбективно) однородное пространство. Тогда для счетного метрического пространства $\left(X, \varrho_{X}\right)$ следующие условия әквивалентны:

(1) $\left(X, \varrho_{X}\right)$ изометрично вкладывается в $\left(Y, \varrho_{Y}\right)$;

(2) $\mathscr{A}_{X} \subseteq \mathscr{A}_{Y}$

(3) для всякой изометрии $f: K \rightarrow Y$ конечного подмножества $X$ существует такая изометрия (в) $F: X \rightarrow Y$, что $f=\left.F\right|_{K}$.

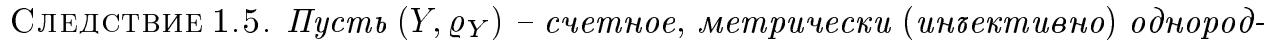
ное пространство. Тогда для метрического пространства $\left(X, \varrho_{X}\right)$ следующие условия әквивалентны:

(1) $\left(X, \varrho_{X}\right)$ изометрично $\left(Y, \varrho_{Y}\right)$;

(2) $X$ счетно, $\mathscr{A}_{X}=\mathscr{A}_{Y}$ и для всяких конечных изометричных подмножеств $A, B \subset X$ существует такая изометрия $($ в) $F: X \rightarrow X$, что $F(A)=B$;

(3) $X$ счетно, $\mathscr{A}_{X} \subseteq \mathscr{A}_{Y}$ и $X \in \operatorname{AME}\left(\mathscr{A}_{Y}\right)$;

(4) для всякой изометрии $h: K \rightarrow Y$ конечного подмножества $X$ существует такая изометрия на $H: X \mapsto Y$, что $h=\left.H\right|_{K}$.

ДоКАЗАТЕЛЬСТВО. ИмПликации $(1) \Rightarrow(2)$ и $(4) \Rightarrow(1)$ очевидны.

$(2) \Rightarrow(3)$. Пусть $f: A \rightarrow X$ - изометрия подмножества конечного подмножества $K \subset Y$. Пусть $G=\operatorname{Iso}(A)$ - конечная группа изометрий $A$. Для всякого элемента $h \in G$ фиксируем такую изометрию $\widetilde{h}: Y \rightarrow Y$, что $\left.\widetilde{h}\right|_{A}=h$. Рассмотрим метрическое пространство $Z=\bigcup_{h \in G} \widetilde{h}(K) \subseteq Y$ и его изометрическое вложение $j: Z \rightarrow X$. Согласно условию можно считать, что $j(A)=f(A)$. В качестве искомого продолжения возьмем $F=\left.j \circ \widetilde{h}\right|_{K}$, где $h=j^{-1} \circ f$.

$(3) \Rightarrow(4)$. Занумеруем точки множеств $X \backslash K=\left\{x_{1}, \ldots, x_{n}, \ldots\right\}$ и $Y \backslash h(K)=$ $\left\{y_{1}, \ldots, y_{n}, \ldots\right\}$. Индукцией по $n$ построим теперь такие конечные множества $K_{n} \subset X$ 
и изометрии $h_{n}: K_{n} \rightarrow Y$, что $K_{n-1} \subseteq K_{n},\left.h_{n}\right|_{K_{n-1}}=h_{n-1}, x_{n} \in K_{n}$ и $h_{n}\left(K_{n}\right) \ni y_{n}$ (мы считаем, что $K_{0}=K$ и $h_{0}=h$ ).

Пусть множество $K_{n-1}$ и изометрия $h_{n-1}: K_{n-1} \rightarrow Y$ уже построены. Рассмотрим множество $\widetilde{K}_{n}=K_{n-1} \cup\left\{x_{n}\right\}$. Если $x_{n} \in K_{n-1}$, то изометрия $\widetilde{h}_{n}: \widetilde{K}_{n} \rightarrow Y$ уже определена, в противном случае такая изометрия существует согласно лемме 1.1. Если $y_{n} \in \widetilde{h}_{n}\left(\widetilde{K}_{n}\right)$, то все сделано; в противном случае согласно лемме 1.1 сушествует такая изометрия $g_{n}: \widetilde{h}_{n}\left(\widetilde{K}_{n}\right) \cup\left\{y_{n}\right\} \rightarrow X$, что $\left.g_{n}\right|_{\widetilde{h}_{n}\left(\widetilde{K}_{n}\right)}=\widetilde{h}_{n}^{-1}$. Теперь полагаем $K_{n}=\widetilde{K}_{n} \cup\left\{g_{n}\left(y_{n}\right)\right\}$ и $h_{n}=g_{n}^{-1}$. Совокупность изометрических вложений $h_{n}$ корректно определяет такое изометрическое отображение на $H: X \mapsto Y$, что $\left.H\right|_{K}=h$.

СлЕДСТВИЕ 1.6. Пусть $\left(Y, \varrho_{Y}\right)$ - полное, метрически (инбективно) однородное пространство. Тогда для метрического пространства $\left(X, \varrho_{X}\right)$ следующие условия эквивалентнь:

(1) $\left(X, \varrho_{X}\right)$ изометрично вкладывается в $\left(Y, \varrho_{Y}\right)$;

(2) $\mathscr{A}_{X} \subseteq \mathscr{A}_{Y}$

(3) для всякой изометрии $f: K \rightarrow Y$ конечного подмножества $X$ существует такая изометрия $(\boldsymbol{\theta}) F: X \rightarrow Y$, что $f=\left.F\right|_{K}$.

СлЕДСТВИЕ 1.7. Пусть $\left(Y, \varrho_{Y}\right)$ - полное, метрически (инбективно) однородное пространство. Тогда для метрического пространства $\left(X, \varrho_{X}\right)$ следующие условия әквивалентны:

(1) $\left(X, \varrho_{X}\right)$ изометрично $\left(Y, \varrho_{Y}\right)$;

(2) $X$ полно, $\mathscr{A}_{X}=\mathscr{A}_{Y}$ и для всяких конечных изометричных подмножеств $A, B \subset X$ существует такая изометрия $($ в) $F: X \rightarrow X$, что $F(A)=B$;

(3) $X$ полно, $\mathscr{A}_{X} \subseteq \mathscr{A}_{Y}$ и $X \in \operatorname{AME}\left(\mathscr{A}_{Y}\right)$;

(4) для всякой изометрии $h: K \rightarrow Y$ конечного подмножества $X$ существует такая изометрия на $H: X \mapsto Y$, что $h=\left.H\right|_{K}$.

ЗАмЕчАниЕ 1.8. Если $\varrho_{Y}$ является ультраметрикой, то в условиях (3) следствий 1.4 и 1.6 и (4) следствий 1.5 и 1.7 можно считать, что $K$ - компактное подмножество [27]. Следствия 1.5 и 1.7 показывают, что в случаях счетных и полных метрических пространств метрическая инъективная однородность эквивалентна метрической сюръективной однородности. Ясно, что леммы 1.1 и 1.3 дают критерий метрической однородности счетного и полного метрических пространств соответственно. Причем все может быть сформулировано для произвольной мощности, что представляет некоторый интерес в связи с аксиоматикой Биркгофа.

ПРЕДЛОЖЕНИЕ 1.9. Метрическое пространство $\left(Y, \varrho_{Y}\right)$ мощности $\lambda$ метрически (инбективно) однородно относительно подмножеств мощности $<\lambda$ тогда и только тогда, когда оно является абсолютным метрическим экстензором для класса всех своих подмножсеств мощности $<\lambda$.

ПРЕДЛОЖЕНИЕ 1.9' . Полное метрическое пространство $\left(Y, \varrho_{Y}\right)$ веса $\lambda$ метрически (инбективно) однородно относительно подмножеств мощности $<\lambda$ тогда и только тогда, когда оно является абсолютным метрическим әкстензором для класса всех своих подмножсеств мощности $<\lambda$.

В случае компактных пространств задача изометричности решается без предположения метрической однородности. 
ПРЕДЛОЖЕНИЕ 1.10 [31; теорема 60.2]. Пусть $\left(Y, \varrho_{Y}\right)$ - компактное метрическое пространство. Тогда для метрического пространства $\left(X, \varrho_{X}\right)$ следующие условия эквивалентны:

(1) $\left(X, \varrho_{X}\right)$ изометрично вкладьввается в $\left(Y, \varrho_{Y}\right)$;

(2) $\mathscr{A}_{X} \subseteq \mathscr{A}_{Y}$.

ПРЕДЛОЖЕНИЕ 1.11. Пусть $\left(Y, \varrho_{Y}\right)$ u $\left(X, \varrho_{X}\right)$ - компактные метрические пространства. Тогда следующие условия әквивалентны:

(1) $\left(X, \varrho_{X}\right)$ изометрично $\left(Y, \varrho_{Y}\right)$;

(2) $\mathscr{A}_{X}=\mathscr{A}_{Y}$.

ДокАЗАТЕЛЬСТво. Импликащия $(1) \Rightarrow(2)$ очевидна.

$(2) \Rightarrow(1)$. Согласно предложению 1.10 существуют изометрические вложения $f: X \rightarrow Y$ и $g: Y \rightarrow X$. Тогда $g \circ f: X \hookrightarrow X$ - изометрическое самовложение, поэтому это есть изометрия на. Следовательно, как $f$, так и $g$ являются изометриями на.

ЗАмЕчАНИЕ 1.12. В предложениях 1.10 и 1.11, т.е. без предположения метрической однородности, нельзя утверждать о возможности продолжения заданной изометрии. Однако в “ортогональном" классе пространств также возможно продолжение заданных изометрий. Метрическое пространство $\left(Y, \varrho_{Y}\right)$ называется метрически жестким [32], если никакое расстояние не принимается дважды. Очевидно, что всякое подмножество мощности $\geqslant 3$ метрически жесткого пространства вкладывается в это пространство единственньм образом, поэтому метрически жесткое пространство является абсолютньм метрическим экстензором для класса всех своих подмножеств мощности $\geqslant 3$. Это означает, что в указанных предположениях аналог леммы 1.1 верен для любого подмножества $K$ мошности $\geqslant 3$. Поэтому (счетное, полное) метрическое пространство $\left(X, \varrho_{X}\right)$ изометрично метрически жесткому (счетному, полному) пространству $\left(Y, \varrho_{Y}\right)$ тогда и только тогда, когда $\mathscr{A}_{X}=\mathscr{A}_{Y}$. Аналогично, такое пространство $\left(X, \varrho_{X}\right)$ изометрично вкладьвается в метрически жесткое (счетное, полное) пространство $\left(Y, \varrho_{Y}\right)$ тогда и только тогда, когда $\mathscr{A}_{X} \subseteq \mathscr{A}_{Y}$.

В замечании 1.8 и работе [27] отмечается метрическая однородность ультраметрических пространств относительно класса компактных подмножеств. В [28] доказана компактная однородность пространства Урысона $U$. Однако, как показьвает пример 3.4, привести обшие свойства, гарантируюшие компактную однородность, довольно сложно.

Итак, (счетное, полное) метрически однородное пространство $Y$ полностью определяется своими конечными подмножествами, т.е. классом $\mathscr{A}_{Y}$. В действительности, во многих случаях весь класс $\mathscr{A}_{Y}$ является “избыточным". Так, знаменитая теорема Менгера утверждает, что если всякое $(n+3)$-точечное подмножество метрического пространства $X$ изометрически вкладьвается в $\mathbb{R}^{n}$, то и все пространство $X$ изометрически вкладьвается в $\mathbb{R}^{n}$. Аналогичная теорема верна и для $n$-мерного пространства Лобачевского. Причем Менгер нашел критерий изометрической вложимости $k$-точечного метрического пространства в $\mathbb{R}^{n}$, сформулированньй целиком в терминах $C_{k}^{2}$ попарных расстояний. Этот критерий заключается в неотрищательности некоторых определителей из квадратов попарных расстояний. Так как соответствуюшие определители пропорциональны квадратам объема, то их естественно рассматри- 
вать как многомерные аналоги формулы Герона. Шеринг и Мэнсон нашли аналогичный критерий изометрической вложимости $k$-точечного метрического пространства в $n$-мерное пространство Лобачевского, а Блюменталь решил соответствующую задачу в сфферической и эллиптической геометриях [31; теоремы 40.1, 111.2, 63.1, 78.2]. Возможно, что было бы интересно решить аналогичную задачу для остальных неприводимых симметрических многообразий ранга 1 . Из универсальности пространств $U$ и $U_{0}$ вытекает, что вложимость в них определяется трехточечными подмножествами (уже в классе симметрик). Блюменталь описал "минимальные" определяющие подмножества $\mathscr{A}_{Y}$ для некоторых (не обязательно метрически однородных) подмножеств плоскости [31; теорема 61.3].

С. Д. Илиадис предложил критерий изометрической вложимости заданного семейства (компактных) метрических пространств в единое компактное метрическое пространство [33; предпоследний абзац]. Этот критерий можно рассматривать как своеобразную топологическую форму теоремы компактности М. Громова [34], [35; леммa 22.6.4].

Для семейства Ж метрических пространств существует компактное метрическое пространство, изометрически содержащее каждое из пространств семейства $\mathscr{K}$, тогда и только тогда, когда диаметры всех пространств семейства $\mathscr{K}$ ограничень сверху некоторой константой $d_{0}$ и для всякого $\varepsilon>0$ существует такая константа $N(\varepsilon)$, что всякое пространство семейства Жू имеет в-сеть мощности не более $N(\varepsilon)$.

2. Склейка метрик. В этой части работы мы приводим примеры различных классов метрик, которые можно склеивать, что вместе с конструкцией Урысона позволяет строить для этих классов метрик универсальные пространства. Далее $X$ и $Y$ - подмножества некоторого множества и на них заданы такие симметрики $\varrho_{1}$ и $\varrho_{2}$ соответственно, что $\left.\varrho_{1}\right|_{Z}=\left.\varrho_{2}\right|_{Z}$, где $Z=X \cap Y$. Мы всегда предполагаем, что $Z$ замкнуто как в $X$, так и в $Y$, т.е. расстояние от каждой точки $(X \cup Y) \backslash Z$ до $Z$ положительно. Следуюшая теорема в случае конечных пространств имеется в [36].

ТеОрема 2.1. Если $\varrho_{1}$ и $\varrho_{2}$ - метрики, то на $X \cup Y$ существует такая метрика $\varrho$, что $\left.\varrho\right|_{X}=\varrho_{1}$ u $\left.\varrho\right|_{Y}=\varrho_{2}$.

ДоказАтельство. Случай 1 . Пусть $Z \neq \varnothing$. Рассмотрим на $(X \cup Y)^{2}$ функцию, которая на $X^{2}$ и $Y^{2}$ совпадает с $\varrho_{1}$ и $\varrho_{2}$ соответственно, а для остальных пар точек определяется формулой

$$
\varrho(x, y)=\min _{z \in Z}\left\{\varrho_{1}(x, z)+\varrho_{2}(z, y)\right\}
$$

Несложно проверить, что построенная функция определена корректно и согласована с $\varrho_{1}$ и $\varrho_{2}$. Проверим, что она действительно является метрикой, т.е. удовлетворяет неравенству треугольника.

Если все три точки $A, B, C$ лежат в одном слагаемом, то все верно. Теперь без ограничения общности можно считать, что $A \in X$ и $B, C \in Y$. Ясно, что нужно проверить справедливость двух неравенств

(1) $\varrho(A, C) \leqslant \varrho(A, B)+\varrho(B, C)$ и

(2) $\varrho(B, C) \leqslant \varrho(B, A)+\varrho(A, C)$. 
(1). Для всякого $\varepsilon>0$ существует такая точка $D_{\varepsilon} \in Z$, что

$$
\begin{aligned}
\varrho(A, B)+\varrho(B, C) & \geqslant \varrho_{1}\left(A, D_{\varepsilon}\right)+\varrho_{2}\left(D_{\varepsilon}, B\right)-\varepsilon+\varrho_{2}(B, C) \\
& \geqslant \varrho_{1}\left(A, D_{\varepsilon}\right)+\varrho_{2}\left(D_{\varepsilon}, C\right)-\varepsilon \geqslant \varrho(A, C)-\varepsilon .
\end{aligned}
$$

Произвольность числа $\varepsilon$ доказьвает нужное неравенство (1).

(2). Для всякого $\varepsilon>0$ сушествуют такие точки $D_{\varepsilon} \in Z$ и $E_{\varepsilon} \in Z$, что

$$
\begin{aligned}
\varrho(B, A)+\varrho(A, C) & \geqslant \varrho_{2}\left(B, D_{\varepsilon}\right)+\varrho_{1}\left(D_{\varepsilon}, A\right)-\varepsilon+\varrho_{1}\left(A, E_{\varepsilon}\right)+\varrho_{2}\left(E_{\varepsilon}, C\right)-\varepsilon \\
& \geqslant \varrho_{2}\left(B, D_{\varepsilon}\right)+\varrho_{1}\left(D_{\varepsilon}, E_{\varepsilon}\right)+\varrho_{2}\left(E_{\varepsilon}, C\right)-2 \varepsilon \\
& =\varrho_{2}\left(B, D_{\varepsilon}\right)+\varrho_{2}\left(D_{\varepsilon}, E_{\varepsilon}\right)+\varrho_{2}\left(E_{\varepsilon}, C\right)-2 \varepsilon \\
& \geqslant \varrho_{2}(B, C)-2 \varepsilon=\varrho(B, C)-2 \varepsilon .
\end{aligned}
$$

Произвольность числа $\varepsilon$ доказьвает нужное неравенство (2).

Случай 2. Пусть $Z=\varnothing$. Выделим в $X$ и $Y$ по точке $x_{*}$ и $y_{*}$ соответственно и расстояние между ними зададим произвольным образом. Теперь положим

$$
\varrho(x, y)=\varrho_{1}\left(x, x_{*}\right)+\varrho\left(x_{*}, y_{*}\right)+\varrho_{2}\left(y_{*}, y\right) .
$$

Согласно случаю 1 формула $\left(+^{\prime}\right)$ задает метрику как на $X \cup\left\{y_{*}\right\}$, так и на $\left\{x_{*}\right\} \cup Y$. Применяя опять случай 1 к множествам $X \cup\left\{y_{*}\right\}$ и $\left\{x_{*}\right\} \cup Y$, мы получаем, что формула $\left(+^{\prime}\right)$ задает метрику на $X \cup Y$.

ТеОРема 2.2. Если $\varrho_{1}$ и $\varrho_{2}$ - ультраметрики, то на $X \cup Y$ существует такая ультраметрика $\varrho$, что $\left.\varrho\right|_{X}=\left.\varrho_{1} u \varrho\right|_{Y}=\varrho_{2}$.

ДоказАтельство. Случай 1 . Пусть $Z \neq \varnothing$. Рассмотрим на $(X \cup Y)^{2}$ функцию, которая на $X^{2}$ и $Y^{2}$ совпадает с $\varrho_{1}$ и $\varrho_{2}$ соответственно, а для остальных пар точек определяется формулой

$$
\varrho(x, y)=\min _{z \in Z} \max \left\{\varrho_{1}(x, z), \varrho_{2}(z, y)\right\}
$$

Несложно проверить, что построенная функция определена корректно и согласована с $\varrho_{1}$ и $\varrho_{2}$. Проверим, что она действительно является ультраметрикой, т.е. удовлетворяет сильному неравенству треугольника.

Если все три точки $A, B, C$ лежат в одном слагаемом, то все верно. Теперь без ограничения общности можно считать, что $A \in X$ и $B, C \in Y$. Ясно, что нужно проверить справедливость двух неравенств

(1) $\varrho(A, C) \leqslant \max \{\varrho(A, B), \varrho(B, C)\}$ и

(2) $\varrho(B, C) \leqslant \max \{\varrho(B, A), \varrho(A, C)\}$.

(1). Для всякого $\varepsilon>0$ существует такая точка $D_{\varepsilon} \in Z$, что

$$
\begin{aligned}
\varrho(A, C) & \leqslant \max \left\{\varrho_{1}\left(A, D_{\varepsilon}\right), \varrho_{2}\left(D_{\varepsilon}, C\right)\right\} \\
& \leqslant \max \left\{\varrho_{1}\left(A, D_{\varepsilon}\right), \max \left\{\varrho_{2}\left(D_{\varepsilon}, B\right), \varrho_{2}(B, C)\right\}\right\} \\
& =\max \left\{\varrho_{1}\left(A, D_{\varepsilon}\right), \varrho_{2}\left(D_{\varepsilon}, B\right), \varrho_{2}(B, C)\right\} \\
& =\max \left\{\max \left\{\varrho_{1}\left(A, D_{\varepsilon}\right), \varrho_{2}\left(D_{\varepsilon}, B\right)\right\}, \varrho_{2}(B, C)\right\} \\
& \leqslant \max \left\{\varrho(A, B)+\varepsilon, \varrho_{2}(B, C)\right\} \\
& \leqslant \max \{\varrho(A, B), \varrho(B, C)\}+\varepsilon .
\end{aligned}
$$


Произвольность числа $\varepsilon$ доказьвает нужное неравенство (1).

(2). Для всякого $\varepsilon>0$ существуют такие точки $D_{\varepsilon} \in Z$ и $E_{\varepsilon} \in Z$, что

$$
\begin{aligned}
\varrho(B, C)=\varrho_{2}(B, C) & \leqslant \max \left\{\varrho_{2}\left(B, D_{\varepsilon}\right), \varrho_{1}\left(D_{\varepsilon}, E_{\varepsilon}\right), \varrho_{2}\left(E_{\varepsilon}, C\right)\right\} \\
& \leqslant \max \left\{\varrho_{2}\left(B, D_{\varepsilon}\right), \varrho_{1}\left(D_{\varepsilon}, A\right), \varrho_{1}\left(A, E_{\varepsilon}\right), \varrho_{2}\left(E_{\varepsilon}, C\right)\right\} \\
& =\max \left\{\max \left\{\varrho_{2}\left(B, D_{\varepsilon}\right), \varrho_{1}\left(D_{\varepsilon}, A\right)\right\}, \max \left\{\varrho_{1}\left(A, E_{\varepsilon}\right), \varrho_{2}\left(E_{\varepsilon}, C\right)\right\}\right\} \\
& \leqslant \max \{\varrho(B, A), \varrho(A, C)\}+\varepsilon .
\end{aligned}
$$

Произвольность числа $\varepsilon$ доказьвает нужное неравенство (2).

Случай 2. Пусть $Z=\varnothing$. Выделим в $X$ и $Y$ по точке $x_{*}$ и $y_{*}$ соответственно и расстояние между ними зададим произвольным образом. Теперь положим

$$
\varrho(x, y)=\max \left\{\varrho_{1}\left(x, x_{*}\right), \varrho\left(x_{*}, y_{*}\right), \varrho_{2}\left(y_{*}, y\right)\right\} .
$$

Согласно случаю 1 формула $\left(\max ^{\prime}\right)$ задает ультраметрику как на $X \cup\left\{y_{*}\right\}$, так и на $\left\{x_{*}\right\} \cup Y$. Применяя опять случай 1 к множествам $X \cup\left\{y_{*}\right\}$ и $\left\{x_{*}\right\} \cup Y$, мы получаем, что формула $\left(\max ^{\prime}\right)$ задает ультраметрику на $X \cup Y$.

В обзоре [37] рассматриваются различные классы метрик. Получить для них теоремы склейки предъявлением аналогов формул $(+)$ и $(\max )$, по-видимому, очень сложно. Однако такие теоремы иногда можно получить, “двигаясь в обратном направлении”, - из существования однородного универсального пространства.

ТеОрема 2.3. Для сепарабельного метрического пространства $(X, \varrho)$ следующие условия әквивалентны:

(1) $(X, \varrho)$ удовлетворяет гипернеравенству 4 точек, т.е.

$$
\varrho(A, B)+\varrho(C, D) \leqslant \max \{\varrho(A, C)+\varrho(B, D), \varrho(A, D)+\varrho(B, C)\} \quad \forall A, B, C, D \in X ;
$$

(2) существуют $\mathbb{R}$-дерево $Y$ и изометрическое вложсение $h: X \hookrightarrow Y$.

Причем минимальное такое $\mathbb{R}$-дерево единственно.

ДокАЗАТЕльство. Так как внутренняя метрика всякого дерева, т.е. метрика $\mathbb{R}$-дерева, удовлетворяет гипернеравенству 4 точек [38]-[42], то импликация $(2) \Rightarrow(1)$ очевидна.

$(1) \Rightarrow(2)$. Фиксируем в пространстве $(X, \varrho)$ счетное всюду плотное подмножество $\left\{A_{i}\right\}_{i=1}^{\infty}$. Для всякого $n$ сушествуют $\mathbb{R}$-дерево $Y_{n}$ и изометрическое вложение $h_{n}: \bigcup_{i=1}^{n} A_{i} \hookrightarrow Y_{n}[39]-[42]$. Мы будем считать, что дерево $Y_{n}$ минимально. Из единственности минимального дерева [38], [39; теорема 3] вытекает, что дерево $Y_{n}$ естественным образом можно считать поддеревом $Y_{n+1}$. Объединение $Y_{\infty}=\bigcup_{i=1}^{\infty} Y_{i}$ возрастаюшей цепочки $\mathbb{R}$-деревьев является $\mathbb{R}$-деревом. Пополнение $Y \mathbb{R}$-дерева $Y_{\infty}$ является $\mathbb{R}$-деревом [43]. Изометрии $h_{n}: \bigcup_{i=1}^{n} A_{i} \hookrightarrow Y_{n} \hookrightarrow Y_{\infty} \hookrightarrow Y$ порождают изометрию $h_{\infty}: \bigcup_{i=1}^{\infty} A_{i} \hookrightarrow Y$, которая равномерно непрерывна и поэтому продолжается до изометрии $h: X \hookrightarrow Y$.

Пусть теперь $h^{\prime}: X \hookrightarrow Y^{\prime}$ - какая-то изометрия в $\mathbb{R}$-дерево $Y^{\prime}$. Из единственности конечного $\mathbb{R}$-дерева вытекает существование согласованных изометрий $h_{n}^{\prime}: Y_{n} \hookrightarrow Y^{\prime}$, которые порождают изометрию $h^{\prime}: Y \hookrightarrow Y^{\prime}$. Построенная изометрия $h^{\prime}: Y \hookrightarrow Y^{\prime}$ приводит к единственности минимального $\mathbb{R}$-дерева. 
Метрические пространства, удовлетворяющие гипернеравенству 4 точек, т.е. $\mathbb{R}$-деревья, играют важную роль в асимптотической геометрии. Так, Громов доказал [44], [45], что все асимптотические конусы и подконусы гиперболических пространств являются вещественными деревьями. $\mathbb{R}$-деревья естественным образом возникают в теории гиперболических групп, в многомерном обобщении теорем Тёрстона о компактификации пространства Тейхмюллера и в теории континуумов [46]-[51]. В [52] доказано, что асимптотический конус метрического пространства, однородного относительно одноточечных подмножеств, также однороден относительно одноточечных подмножеств. Асимптотический конус плоскости Лобачевского (пример 3.6) и континуальный аналог теоремы 4.3 показьвают, что в сформулированном утверждении одноточечные подмножества нельзя заменить на произвольные. Вопрос о справедливости аналога теоремы Каповича-Лииба для однородности относительно конечных подмножеств представляется открытьм.

Теорема 2.4. Если $\varrho_{1}$ и $\varrho_{2}$ - метрики, удовлетворяющие гипернеравенству

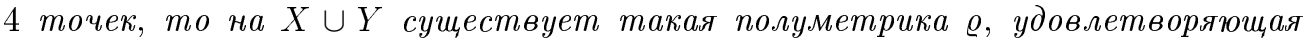
гипернеравенству 4 точек, что $\left.\right|_{X}=\varrho_{1}$ и $\left.\varrho\right|_{Y}=\varrho_{2}$.

ДоказАТЕльство. Пусть $X^{*}$ и $Y^{*}$ - соответствующие объемлющие $\mathbb{R}$-деревья. Через $Z_{X}^{*}$ и $Z_{Y}^{*}$ обозначим минимальные поддеревья в $X^{*}$ и $Y^{*}$, содержашие $Z$. $\mathbb{R}$-деревья $Z_{X}^{*}$ и $Z_{Y}^{*}$ изометричны. Результат склейки $\mathbb{R}$-деревьев $X^{*}$ и $Y^{*}$ по поддеревьям $Z_{X}^{*}$ и $Z_{Y}^{*}$ является $\mathbb{R}$-деревом, содержащим образ $X \cup Y$. При этом, однако, некоторые точки $X$ и $Y$ могут оказаться в $Z^{*}$ и даже склеиться. Поэтому на $X \cup Y$ может индуцироваться не метрика, а полуметрика.

Мы говорим, что метрика $\varrho$ на $X$ является евклидовой (гильбертовой), если $(X, \varrho)$ изометрически вкладьвается $h: X \hookrightarrow \mathbb{R}^{n}$ в некоторое евклидово пространство $\mathbb{R}^{n}$ (в гильбертово пространство $\ell_{2}$ ).

ТеОРема 2.5. Если $\varrho_{1}$ и $\varrho_{2}-$ евклидовы метрики, то на $X \cup Y$ существует такая евклидова полуметрика $\varrho$, что $\left.\varrho\right|_{X}=\varrho_{1}$ и $\left.\right|_{Y}=\varrho_{2}$.

ДокАЗАТЕльство. Пусть $h_{X}: X \hookrightarrow \mathbb{R}^{n}$ и $h_{Y}: Y \hookrightarrow \mathbb{R}^{m}$ - изометрические вложения. Без ограничения обшности можно считать, что $n=m$. Согласно метрической однородности пространства $\mathbb{R}^{n}$ относительно всех подмножеств можно считать, что эти вложения совпадают на $Z$. Таким образом, корректно определено отображение $h_{X} \cup h_{Y}: X \cup Y \rightarrow \mathbb{R}^{n}$, задаюшее на $X \cup Y$ евклидову полуметрику.

ТеОрема 2.5'. Если $\varrho_{1}$ и $\varrho_{2}$ - вполне ограниченные гильбертовы метрики, то на $X \cup Y$ существует такая гильбертова полуметрика $\varrho$, что $\left.\varrho\right|_{X}=\varrho_{1} u$ $\left.\varrho\right|_{Y}=\varrho_{2}$.

Напомним, что под сим.метрикой на множестве $X$ понимается такое отображение $\varrho: X \times X \rightarrow[0, \infty)$, что

(1) $\varrho(A, B)=\varrho(B, A) \forall A, B \in X$;

(2) $\varrho(A, B)=0 \Leftrightarrow A=B \forall A, B \in X$.

Отображение $\varphi: X \rightarrow \mathbb{Z}$ называется финитньм, если множество $\operatorname{supp} \varphi=\{A \in X$ : $\varphi(A) \neq 0\}$ конечно. Для всякой финитной функции $\varphi$ определена сумма $\Sigma \varphi=$ $\sum_{A \in X} \varphi(A)$ и норма $|\varphi|=\sum_{A \in X}|\varphi(A)|$. 
Говорят [53], [37], что симметрика имеет отрицательный тип, если для всякой финитной функшии $\varphi$ с нулевой суммой $\Sigma \varphi=0$ имеет место неравенство

$$
\sum_{A, B \in X} \varphi(A) \varphi(B) \varrho(A, B) \leqslant 0
$$

Важно отметить, что симметрика отрицательного типа не обязана быть метрикой, но “близка" к метрике. В теории метрических пространств важное значение имеет преобразование симметрики $[31 ; \S 52]$.

Для симметрического пространства $(X, \varrho)$ следующие условия әквивалент$H b l$.

(1) Симметрика $\varrho$ удовлетворяет неравенству $(\varphi)$ для всякой финитной функиии $\varphi$ с нулевой суммой $\Sigma \varphi=0$ и трехточечным носителем $|\operatorname{supp} \varphi|=3$.

(2) Симметрика $\varrho^{1 / 2}$ является метрикой.

Из теоремы Шенберга [31; с. 107, следствие] (восходящей еще к Кэли) о том, что симметрика @ имеет отрицательный тип тогда и только тогда, когда метри$\kappa a \varrho^{1 / 2}$ является гильбертовой, немедленно вытекает, что теорему $2.5^{\prime}$ можно сформулировать в следующем эквивалентном виде.

СлЕДСТВИЕ 2.6. Если $\varrho_{1}$ и $\varrho_{2}$ - вполне ограниченные симметрики отрицательного типа, то на $X \cup Y$ существует такая полусимметрика отрицательного типа $\varrho$, что $\left.\varrho\right|_{X}=\varrho_{1}$ и $\left.\varrho\right|_{Y}=\varrho_{2}$.

ДОКАЗАТЕЛЬСТВО ТЕОРЕМЫ 2.5'. Пусть $h_{X}: X \hookrightarrow \ell_{2}$ и $h_{Y}: Y \hookrightarrow \ell_{2}-$ изометрические вложения.

Шаг 1. Пусть множество $Z$ конечно. Согласно метрической однородности пространства $\ell_{2}$ относительно всех конечных подмножеств можно считать, что эти вложения совпадают на $Z$. Таким образом, корректно определено отображение $h_{X} \cup h_{Y}$ : $X \cup Y \rightarrow \ell_{2}$, задающее на $X \cup Y$ гильбертову полуметрику.

Шаг 2. Пусть множество $Z$ бесконечно. Фиксируем в нем счетное всюду плотное подмножество $\left\{z_{i}\right\}_{i=1}^{\infty}$. Для всякого $k \geqslant 2$ с помошю шага 1 на множестве $X \sqcup_{Z_{k}} Y$, где $Z_{k}=\left\{z_{i}\right\}_{i=1}^{k}$, определяется некоторая гильбертова полуметрика $\varrho_{k}$. Покажем, что из последовательности полуметрик $\left\{\varrho_{k}\right\}_{k=2}^{\infty}$ можно выбрать сходящуюся подпоследовательность.

На множестве $X \sqcup Y$ введем метрику, о которой говорится в доказательстве теоремы 2.1 (случай 2$),$ с $\varrho\left(x_{*}, y_{*}\right)=1$. На квадрате $(X \sqcup Y)^{2}$ введем тах-метрику. Теперь несложно проверить, что все функции $\left\{\varrho_{k}\right\}_{k=2}^{\infty}$ равностепенно непрерьвны с $\delta=\varepsilon / 2$ для всякого $\varepsilon<1$. Из теоремы Арцела-Асколи с помощњю диагонального процесса по ограниченным множествам получается поточечно сходящаяся последовательность $\left\{\varrho_{k_{i}}\right\}_{i=1}^{\infty}$. Предельная функция $\varrho$ задает корректно определенную на $(X \cup Y)^{2}$ функцию, являюшуюся полуметрикой на $X \cup Y$. Гильбертовость построенной полуметрики $\varrho$ можно проверить с помошью детерминантного критерия Кэли-Менгера [31; лемма 53.1, §40] или с помощью сформулированного выше критерия Шенберга. 
ЗАмечАниЕ 2.7. В некоторых случаях продолжение на $X \cup Y$ определено однозначно и неизбежно принимает нулевое значение для некоторых пар различных точек. Рассмотрим два множества $X=\{A, B, C\}$ и $Y=\{A, B, D\}$ с метриками $\varrho_{1}(A, C)=$ $\varrho_{1}(B, C)=\varrho_{2}(A, D)=\varrho_{2}(B, D)=1$ и $\varrho_{1}(A, B)=\varrho_{2}(A, B)=2$. Тогда для всякой евклидовой полуметрики (полусимметрики, удовлетворяющей гипернеравенству 4 точек) на $X \cup Y$ обязательно $\varrho(C, D)=0$.

3. Существование. В этой части работы мы приводим различные примеры метрически однородных пространств. Все примеры разбиваются на две групшы: (1) конкретные примеры; (2) примеры, получающиеся конструкщией, восходяшей к П. С. Урысону.

ПримеРЫ 3.1-3.3. $n$-мерное евклидово и сферическое пространства $\mathbb{R}^{n}, S^{n}$ и гиперболическое пространство $\mathscr{H}^{n}$ (пространство Лобачевского) с канонической метрикой являются метрически однородными относительно класса всех своих подмножеств $[31 ; \S 38$, свойство IV, $\S 105$, упражнение 3$]$.

ПримеР 3.4. Бесконечномерное сепарабельное гильбертово пространство $\ell_{2}$ метрически однородно относительно класса всех своих конечных подмножеств [31; §53], но не однородно относительно класса счетных дискретных подмножеств (следствие 4.5) и класса счетных компактных подмножеств.

Действительно, рассмотрим в $\ell_{2}$ подмножество $A=\left\{x_{n}\right\}_{n=0}^{\infty}$ : все координаты точки $x_{0}$ равны $0 ;$ у точки $x_{n} n$-я координата равна $2^{-n}$, а все остальные координаты нулевые. Легко проверить, что в индуцированной из $\ell_{2}$ метрике множество $A$ является метрически жестким компактным пространством. Следовательно, всякая изометрия $A$ в себя является тождественным отображением. Пусть теперь $B=\left\{y_{n}\right\}_{n=0}^{\infty}$, где все координаты точки $y_{0}$ равны 0 ; у точки $y_{n}(n+1)$-я координата равна $2^{-n}$, а все остальные координаты нулевые. Легко видеть, что отображение $f: B \mapsto A \subset \ell_{2}$, заданное формулой $f\left(y_{n}\right)=x_{n}$, является изометрией (причем это единственная изометрия множества $B$ в множество $A$ ). Пусть $F: \ell_{2} \rightarrow \ell_{2}$ - такое линейное отображение, что $\left.F\right|_{B}=f$ (согласно теореме Мазура-Улама [54] всякая изометрия является линейным отображением). Тогда образом аффинной оболочки множества $B$ (собственного подпространства $\left.\ell_{2}\right)$ является аффинная оболочка множества $A$, т.е. все пространство $\ell_{2}$. Это означает, что отображение $F$ не является взаимно однозначным $\left(\left|F^{-1}\left(F\left(x_{1}\right)\right)\right| \geqslant 2\right)$ и тем более не является изометрией.

ПримеР 3.5. Пространство $\ell_{2}$, в качестве симметрики на котором берется квадрат гильбертовой метрики, метрически однородно относительно класса всех своих конечных подмножеств и универсально для класса всех сепарабельных симметрик отрицательного типа.

Пример 3.6. Никакое сепарабельное дерево не может быть универсальным метрическим деревом и не может быть даже топологически однородньм. Однако А. Г. Дюбина, И.В. Полтерович и А. Шнирельман нашли [55], [56] явное метрическое представление асимптотического конуса гиперболических пространств Лобачевского, которьй оказался $\mathbb{R}$-деревом континуального веса, универсальным в классе $\mathbb{R}$-деревьев континуального веса. Повторение рассуждений из [55], [56] позволяет показать, что предъявленное там пространство является метрически однородньм не только относительно класса всех одноточечных, но и относительно класса всех конечных подмножеств. 
Рассмотрим теперь следуюшую задачу: описать те классы $\mathscr{K}$, для которых сушествует такое метрически однородное пространство $Y$, что $\mathscr{K}=\mathscr{A}_{Y}$.

ЛЕмма 3.7. Если метрическое пространство (Y, $\left.\varrho_{Y}\right)$ метрически инбективно однородно и для представления конечного множества $K=K_{1} \cup K_{2}$ на $K_{1}$ и $K_{2}$ имеются такие метрики $\varrho_{1}$ и $\varrho_{2}$, что $\left(K_{1}, \varrho_{1}\right),\left(K_{2}, \varrho_{2}\right) \in \mathscr{A}_{Y}$ и $\left.\varrho_{1}\right|_{K_{1} \cap K_{2}}=$ $\left.\varrho_{2}\right|_{K_{1} \cap K_{2}}$, то на $K$ существует такая метрика $\varrho$, что $(K, \varrho) \in \mathscr{A}_{Y}$ и $\left.\right|_{K_{1}}=\varrho_{1}$, $\left.\varrho\right|_{K_{2}}=\varrho_{2}$.

ДОКАЗАТЕЛЬСТвО. Пусть $i_{1}: K_{1} \hookrightarrow Y$ и $i_{2}: K_{2} \hookrightarrow Y$ - изометрические вложения. Из метрической инъективной однородности пространства $Y$ вытекает сушествование такой изометрии (в) $F: Y \rightarrow Y$, что $\left.F\right|_{i_{1}\left(K_{1} \cap K_{2}\right)}=i_{2} \circ i_{1}^{-1}$. Тогда вложение $i: K \hookrightarrow Y$, задаваемое формулой $i(k)=F\left(i_{1}(k)\right)$ для $k \in K_{1}$ и формулой $i(k)=i_{2}(k)$ для $k \in K_{2}$, порождает на $K$ искомую метрику $\varrho=\left.\varrho_{Y}\right|_{i(K)}$.

В некоторых случаях предъявленное условие является характеристическим.

Теорема 3.8. Для счетного семейства К конечных метрических пространств следующие условия эквивалентны:

(1) существует такое счетное метрически однородное пространство $\left(Y, \varrho_{Y}\right)$, что $\mathscr{K}=\mathscr{A}_{Y}$

(2) существует такое счетное метрически инбективно однородное пространство $\left(Y, \varrho_{Y}\right)$, что $\mathscr{K}=\mathscr{A}_{Y}$;

(3) всякое подмножество пространства из К содержится в $\mathscr{K}$ и если для представления конечного множества $K=K_{1} \cup K_{2}$ на $K_{1}$ и $K_{2}$ заданы такие метрики $\varrho_{1}$ и $\varrho_{2}$, ито $\left(K_{1}, \varrho_{1}\right),\left(K_{2}, \varrho_{2}\right) \in \mathscr{K}$ и $\left.\varrho_{1}\right|_{K_{1} \cap K_{2}}=\left.\varrho_{2}\right|_{K_{1} \cap K_{2}}$, то на $K$ существует такая метрика $\varrho$, что $(K, \varrho) \in \mathscr{K}$ и $\left.\right|_{K_{1}}=\varrho_{1}$, $\left.\varrho\right|_{K_{2}}=\varrho_{2}$

(4) всякое подмножество пространства из К содержится в $\mathscr{K}$ и если для представления конечного множества $K=K_{1} \cup K_{2} c\left|K_{1} \backslash K_{2}\right|+\left|K_{2} \backslash K_{1}\right|=2$ на $K_{1}$ и $K_{2}$ заданы такие метрики $\varrho_{1}$ и $\varrho_{2}$, что $\left(K_{1}, \varrho_{1}\right),\left(K_{2}, \varrho_{2}\right) \in \mathscr{K}$ и $\left.\varrho_{1}\right|_{K_{1} \cap K_{2}}=\left.\varrho_{2}\right|_{K_{1} \cap K_{2}}$, то на $K$ существует такая метрика $\varrho$, что $\left.(K, \varrho) \in \mathscr{K} u \varrho\right|_{K_{1}}=\varrho_{1},\left.\varrho\right|_{K_{2}}=\varrho_{2}$.

ДокАЗАТЕЛЬСТво. Импликации $(1) \Rightarrow(2)$ и $(3) \Rightarrow(4)$ очевидны. Импликация $(2) \Rightarrow(3)$ содержится в лемме 3.7, а импликация $(4) \Rightarrow(1)$ доказана в [27].

ПреДЛОЖЕНИЕ 3.9. Пополнение ультраметрического метрически инбективно однородного пространства является метрически инбективно однородным пространством.

ДокАЗАТЕльСТВО. Пусть $(X, \varrho)$ - ультраметрическое метрически инъективно однородное пространство, $Y$ - его пополнение, а $f: C \rightarrow Y$ - изометрия конечного подмножества $Y$. Выделим в $X \backslash C$ счетное всюду плотное подмножество $\left\{x_{1}, \ldots, x_{n}, \ldots\right\}$. Положим $C_{n}=C \cup\left\{x_{1}, \ldots, x_{n}\right\}$ и по индукции построим последовательность продолжаюших друг друга изометрий $f_{n}: C_{n} \rightarrow Y$. Эти изометрии $f_{n}$ корректно зададут изометрию $f_{\infty}: C \cup\left\{x_{1}, \ldots, x_{n}, \ldots\right\} \rightarrow Y$. Так как пространство $Y$ полно, то изометрия $f_{\infty}$ продолжится до изометрии $F: Y \rightarrow Y$.

Полагая $C_{0}=C$ и $f_{0}=f$, можно считать, что база индукции имеется. Проведем индуктивный шаг. Пусть $\varepsilon_{n}=\min \left\{\varrho\left(y_{1}, y_{2}\right): y_{1}, y_{2} \in C_{n}, y_{1} \neq y_{2}\right\}>0$. Так как $Y$ 
является пополнением $(X, \varrho)$, то существуют такие отображения $i_{\varepsilon_{n}}, f_{\varepsilon_{n}}: C_{n-1} \rightarrow X$, что $\varrho\left(i_{n-1}, i_{\varepsilon_{n}}\right)<\varepsilon_{n}$ и $\varrho\left(f_{n-1}, f_{\varepsilon_{n}}\right)<\varepsilon_{n}$, где $i_{n-1}$ обозначает тождественное вложение $C_{n-1}$ в $Y$. Из равнобедренности треугольников в ультраметрическом пространстве несложно вьвести, что отображения $i_{\varepsilon_{n}}$ и $f_{\varepsilon_{n}}$ также являются изометриями. Из метрической инъективной однородности пространства $X$ вытекает существование изометрии $F_{n}: X \rightarrow X$, продолжающей изометрию $f_{\varepsilon_{n}} \circ i_{\varepsilon_{n}}^{-1}$. Искомая изометрия $f_{n}$ совпадает с $f_{n-1}$ на $C_{n-1}$ и $f_{n}\left(x_{n}\right)=F_{n}\left(x_{n}\right)$.

ЗАмЕЧАНИЕ 3.10. Дополнительное рассмотрение обратных отображений (как в следствии 1.5) позволяет снять в доказанном предложении слово “инъективность". Кроме того, как в лемме 1.2, можно получить аналогичное утверждение для метрической однородности относительно класса компактных подмножеств.

Пример 3.11. Пополнение пространства из условия (1) теоремы 3.8 в случае класса всех конечных ультраметрических пространств с рациональными расстояниями определяет однородную ультраметрику на пространстве иррациональных чисел $U_{0}[27]$.

ПримеР 3.12. Пополнение метрически однородного пространства из условия (1) теоремы 3.8 в случае класса всех конечных метрических пространств, удовлетворяюших гипернеравенству 4 точек, с рациональными расстояниями не является метрически однородным (пример 3.6). Однако именно пополнение пространства из условия (1) теоремы 3.8 в случае класса всех конечных метрических пространств с рациональными расстояниями дает универсальное метрическое пространство Урысона $U$ [25].

Пример 3.13. Автор не может утверждать, что всякое полное метрически однородное пространство содержит счетное плотное метрически однородное подпространство, т.е. получается применением теоремы 3.8 и предложения 3.9 к некоторому подходящему классу $\mathscr{K}$.

4. Группа изометрий. В [28], [27] доказано, что всякая изометрия компактного подмножества пространства $U\left(U_{0}\right)$ продолжается до изометрии всего пространства $U\left(U_{0}\right)$. Однако “неканоничность" продолжения не позволяет получать продолжение группового действия. В этой части работы мы приводим различные результаты о возможности продолжения изометрий со специальньми свойствами. Хотя все результаты могут быть сформулированы в аксиоматической форме, мы предпочитаем более простые конкретные формулировки.

ОПРЕДЕЛЕНИЕ 4.1. Клиффордовой транслящиӗ (на расстояние $r$ ) метрического пространства называется такая изометрия, которая перемещает все точки на одно и то же расстояние $(r)$.

ТЕОрема 4.2. Всякое изометрическое действие конечной группьи $G$ на компактном подмножестве $C \subseteq U\left(C \subseteq U_{0}\right)$ пространства Урысона $U\left(U_{0}\right)$ в качестве клиффордовых транслячий на расстояние $r$ можно продолжить до изометрического действия на всем пространстве $U\left(U_{0}\right)$ в качестве клиффордовых транслячий на расстояние $r$.

ДокАЗАТЕЛЬСТВо. С помошњю произвольной точки $x \in U \backslash C$ определим на $C \sqcup G$ метрику, которая совпадает на $C \sqcup e$ с метрикой пространства $U$ и в которой естественное действие групы $G$ на $C \sqcup G$ является действием посредством клиффордовых трансляций на расстояние $r$. 
Именно, положим $\varrho\left(g_{1}, g_{2}\right)=r$ для $g_{1} \neq g_{2}$ и $\varrho(c, g)=\varrho\left(g^{-1} c, x\right)$. Докажем, что так определенное отображение $\varrho:(C \sqcup G)^{2} \rightarrow[0, \infty)$ является метрикой.

Для этого нужно проверить справедливость неравенства треугольника в следующих четырех случаях:

(1) $\varrho\left(c_{1}, c_{2}\right) \leqslant \varrho\left(c_{1}, g\right)+\varrho\left(g, c_{2}\right)$;

(2) $\varrho\left(g_{1}, g_{2}\right) \leqslant \varrho\left(g_{1}, c\right)+\varrho\left(c, g_{2}\right)$;

(3) $\varrho\left(c_{1}, g\right) \leqslant \varrho\left(c_{1}, c_{2}\right)+\varrho\left(c_{2}, g\right)$;

(4) $\varrho\left(c, g_{1}\right) \leqslant \varrho\left(c, g_{2}\right)+\varrho\left(g_{2}, g_{1}\right)$.

(1). $\varrho\left(c_{1}, g\right)+\varrho\left(g, c_{2}\right)=\varrho\left(g^{-1}\left(c_{1}\right), x\right)+\varrho\left(x, g^{-1}\left(c_{2}\right)\right) \geqslant \varrho\left(g^{-1}\left(c_{1}\right), g^{-1}\left(c_{2}\right)\right)=$ (так как $g$ является изометрией) $\varrho\left(c_{1}, c_{2}\right)$.

(2). $\varrho\left(g_{1}, c\right)+\varrho\left(c, g_{2}\right)=\varrho\left(g_{1}^{-1}(c), x\right)+\varrho\left(x, g_{2}^{-1}(c)\right) \geqslant \varrho\left(g_{1}^{-1}(c), g_{2}^{-1}(c)\right)=($ так как $g_{2}$ является изометрией $)\left(g_{2} g_{1}^{-1}(c), c\right)=\left(\right.$ так как $g_{2} g_{1}^{-1}$ является транслящией на расстояние $r) r=\varrho\left(g_{1}, g_{2}\right)$.

(3). $\varrho\left(c_{1}, c_{2}\right)+\varrho\left(c_{2}, g\right)=\varrho\left(c_{1}, c_{2}\right)+\varrho\left(g^{-1}\left(c_{2}\right), x\right)=($ так как $g$ является изометрией $)$ $\varrho\left(g^{-1}\left(c_{1}\right), g^{-1}\left(c_{2}\right)\right)+\varrho\left(g^{-1}\left(c_{2}\right), x\right) \geqslant \varrho\left(g^{-1}\left(c_{1}\right), x\right)=\varrho\left(c_{1}, g\right)$.

(4). $\varrho\left(c, g_{2}\right)+\varrho\left(g_{2}, g_{1}\right)=\varrho\left(g_{2}^{-1}(c), x\right)+r=$ (так как $g_{2}^{-1} g_{1}$ является трансляцией на расстояние $r) \varrho\left(g_{2}^{-1}(c), x\right)+\varrho\left(g_{1}^{-1}(c), g_{2}^{-1}(c)\right) \geqslant \varrho\left(g_{1}^{-1}(c), x\right)=\varrho\left(c, g_{1}\right)$.

Теперь действие $G$ с $C$ на все пространство $U$ продолжается повторением стандартной процедуры рассмотрения счетного всюду плотного подмножества $U \backslash C$, как в доказательстве леммы 1.1 и следствия 1.5 (и в случае бесконечного $C$ опирается на возможность продолжения изометрии с компактного подмножества).

В случае компактного подмножества пространства $U_{0}$ на множестве $C \sqcup G$ возникает ультраметрика, поэтому получается доказательство второго утверждения.

Отсюда автоматически вытекает, что любые две точки как пространства $U$, так и пространства $U_{0}$ можно поменять местами посредством некоторой инволютивной клиффордовой трансляции. В связи с этим напомним проблему ван Дантцига о возможности поменять местами любые две точки многообразия инволюцией [57; с. 104]. Свойство же дизъюнктности дисков (DDP) пространства $U$ [28] можно сформулировать в следующей более сильной форме. Для всякого компакта $C \subset U$ и всякого $\varepsilon>0$ существует такая инволютивная клиффордова трансляиия $\sigma$ на расстояние $\varepsilon$, что $\varrho(c, \sigma(C))=\varepsilon$ для всех точек $c \in C$.

Подмножество $\Delta \subseteq X$ метрического пространства назовем равносторонним, если все попарные расстояния между различными точками $\Delta$ одинаковы. Для равностороннего подмножества $\Delta \subseteq X$ рассмотрим семейство $\mathscr{U}$ всех равносторонних подмножеств $A \subset X$, которые содержат $\Delta$. Семейство $\mathscr{U}$ частично упорядочено, и всякая цепь в $\mathscr{U}$ ограничена сверху (объединением соответствуюших подмножеств). Следовательно, согласно лемме Цорна в $\mathscr{U}$ есть (возможно не единственньй) максимальньй элемент $\widetilde{A}$. Представляется вероятньм, что мошность множества $\widetilde{A} \backslash A$ не зависит от выбора максимального элемента семейства $\mathscr{U}$. Пусть $\Delta_{r}$ означает счетное метрическое пространство, в котором расстояние между любыми двумя различными точками равно $r$.

ТЕОРема 4.3. Если метрическое пространство $Y$ изометрически содержит $\Delta_{r}$ (для некоторого $r$ ), то в $Y$ имеется такое счетное равностороннее подмножество $A$, что для всякого собственного подмножества $B \subsetneq A$ и всякой изометрии $H: Y \rightarrow Y$ (не обязательно на) имеет место $A \backslash H(B) \neq \varnothing$. 
ДокАЗАТЕЛЬСТво. Возьмем подмножество $\Delta_{r} \subset Y$ и рассмотрим некоторое максимальное объемлющее равностороннее множество $A$. Из включения $A \supseteq \Delta_{r}$ вытекает бесконечность множества $A$. С другой стороны, $A$ дискретно (и замкнуто в $Y$ ), поэтому $|A|=\aleph_{0}$. Пусть теперь $A \backslash B \neq \varnothing$ и $H: Y \rightarrow Y$ - изометрия. Тогда $H(A) \backslash H(B) \neq \varnothing$ и если $H(B) \supseteq A$, то цепочка включений $H(A) \supset H(B) \supseteq A$ противоречит максимальности $A$.

ГИПОТЕЗА 4.4. Для любой изометрии $h: A \rightarrow B$ максимальных равносторонних подмножсеств $U$ существует (притом единственная) изометрия на $H: U \mapsto U$ такая, что $\left.H\right|_{A}=h$.

СЛЕДСТВИЕ 4.5. Пространства $\ell_{2}, U$ и $U_{0}$ являются метрически однородными относительно компактных подмножеств, но не являются метрически однородными относительно подмножсеств $\Delta_{r}$ (которые замкнуты и локально компактны).

Приведенное следствие в случае пространства $U$ дает ответ на вопрос из [28] и решает задачу Урысона: “Остается открытым вопрос: нельзя ли любые два конгруэнтных счетных множества в $U$ перевести друг в друга изометрическим отображением пространства $U$ на себя?" Полученное в [28], [29] решение задачи Урысона хуже тем, что в нем счетное множество имеет сложную топологическую структуру, но лучше тем, что оно дает отрицательный ответ не только для изометрий, но и для гомеоморфизмов "на".

ТЕОРема 4.6. Для всякой нормы $\|\cdot\|$ на конечномерном векторном пространстве $V^{n}$ существует такое скалярное произведение $\langle\cdot, \cdot\rangle$, что всякая $\|\cdot\|$-изометрия сохраняет скалярное произведение $\langle\cdot, \cdot\rangle$.

ДоКАЗАТЕЛЬСТВО. Пусть $B=\{x:\|x\| \leqslant 1\}$ - единичньй шар в заданной норме. Рассмотрим $E_{B}$ - эллипсоид Джона вьпуклого тела $B$ [58], [59; лемма 13.2]. Эллипсоид $E_{B}$ задает некоторое скалярное произведение $\langle\cdot, \cdot\rangle$. Пусть теперь $F: V^{n} \rightarrow V^{n}$ - некоторая $\|\cdot\|$-изометрия. По теореме Мазура-Улама отображение $F$ является линейньм [54]. Тогда отображение $F$ меняет объемы (в $\langle\cdot, \cdot\rangle$-метрике) всех фигур в одно и тоже число раз. Из $F(B)=B$ вытекает, что

$$
\operatorname{vol} F\left(E_{B}\right)=\operatorname{vol} E_{B}
$$

Согласно теореме Джона включение $F\left(E_{B}\right) \supseteq F(B)=B$ и равенство (vol) влечет равенство $F\left(E_{B}\right)=E_{B}$. Последнее равенство и означает, что $F$ является $\langle\cdot, \cdot\rangle$-изометрией.

Предположение конечномерности существенно для справедливости доказанной теоремы.

Tеорема 4.7. B пространстве $c=\left\{\mathbf{x}=\left\{x_{n}\right\}_{n=1}^{\infty}: \sup _{n}\left|x_{n}\right|<\infty\right\}$ c sup-нормой существуют три $\|\cdot\|$-изометрии $\varphi_{1}, \varphi_{2}, \varphi_{3}$ и вектор а такие, что $\varphi_{2}(\mathbf{a})=$ $\mathbf{a}+\varphi_{1}(\mathbf{a}), \varphi_{3}(\mathbf{a})=\mathbf{a}-\varphi_{1}(\mathbf{a})$ и для всякого скалярного произведения $\langle\cdot, \cdot\rangle$ на двумерном подпространстве $\mathscr{L}\left(\mathbf{a}, \varphi_{1}(\mathbf{a})\right)$ (на всем пространстве $\left.c\right)\left\langle\varphi_{i}(\mathbf{a}), \varphi_{i}(\mathbf{a})\right\rangle \neq$ $\langle\mathbf{a}, \mathbf{a}\rangle$ для некоторого $i \in\{1,2,3\}$. 
ДокАЗАТЕЛЬСТВО. Возьмем вектор $\mathbf{a}=\left\{x_{n}\right\}_{n=1}^{\infty}$, где

$$
x_{n}=\left\{\begin{array}{lll}
1 & \text { при } n \equiv 1 \bmod 3 \\
0 & \text { при } n \not \equiv 1 \bmod 3 .
\end{array}\right.
$$

Линейную изометрию $\varphi_{1}: c \rightarrow c$ задаем формулой $\varphi_{1}(\mathbf{x})=\mathbf{y}$, где

$$
y_{n}= \begin{cases}x_{n+1} & \text { при } n \equiv 1 \bmod 3 ; \\ x_{n-1} & \text { при } n \equiv 2 \bmod 3 ; \\ x_{n} & \text { при } n \equiv 0 \bmod 3 .\end{cases}
$$

Линейную изометрию $\varphi_{2}: c \rightarrow c$ задаем формулой $\varphi_{2}(\mathbf{x})=\mathbf{y}$, где

$$
y_{n}= \begin{cases}x_{(n+1) / 2} & \text { при } n \equiv 1 \bmod 6 ; \\ x_{n / 2} & \text { при } n \equiv 2 \bmod 6 ; \\ x_{n} & \text { при } n \equiv 0 \bmod 3 ; \\ x_{n / 2} & \text { при } n \equiv 4 \bmod 6 ; \\ x_{(n-1) / 2} & \text { при } n \equiv 5 \bmod 6 .\end{cases}
$$

Линейную изометрию $\varphi_{3}: c \rightarrow c$ задаем формулой $\varphi_{3}(\mathbf{x})=\mathbf{y}$, где

$$
y_{n}= \begin{cases}x_{(n+1) / 2} & \text { при } n \equiv 1 \bmod 6 ; \\ -x_{n / 2} & \text { при } n \equiv 2 \bmod 6 ; \\ x_{n} & \text { при } n \equiv 0 \bmod 3 ; \\ x_{n / 2} & \text { при } n \equiv 4 \bmod 6 ; \\ -x_{(n-1) / 2} & \text { при } n \equiv 5 \bmod 6 .\end{cases}
$$

Из тождества Шварца

$$
\langle\mathbf{a}+\mathbf{b}, \mathbf{a}+\mathbf{b}\rangle+\langle\mathbf{a}-\mathbf{b}, \mathbf{a}-\mathbf{b}\rangle=2(\langle\mathbf{a}, \mathbf{a}\rangle+\langle\mathbf{b}, \mathbf{b}\rangle)
$$

теперь легко получить, что ни для какого скалярного произведения четыре вектора $\mathbf{a}, \mathbf{b}=\varphi_{1}(\mathbf{a}), \mathbf{a}+\mathbf{b}=\varphi_{2}(\mathbf{a})$ и $\mathbf{a}-\mathbf{b}=\varphi_{3}(\mathbf{a})$ не могут иметь одинаковую ненулевую длину.

СлЕДСТвИЕ 4.8. Если конечномерное нормированное векторное пространство метрически однородно по отношению $к$ классу всех двухточечных подмножеств, то оно евклидово.

ДоКАЗАТЕЛЬСТво. Пусть $\langle\cdot, \cdot\rangle-$ это скалярное произведение, о котором говорится в теореме 4.6. Возьмем некоторьй ненулевой вектор а и пронормируем скалярное произведение таким образом, чтобы $\|\mathbf{a}\|^{2}=\langle\mathbf{a}, \mathbf{a}\rangle$. Пусть теперь $\mathbf{b}-$ произвольный вектор. Рассмотрим вектор $\mathbf{c}=\frac{\|\mathbf{a}\|}{\|\mathbf{b}\|} \mathbf{b}$. Тогда $\|\mathbf{c}\|=\|\mathbf{a}\|$, поэтому по свойствам нормы $\|\cdot\|$ существует такая $\|\cdot\|$-изометрия $F$, что $F(\mathbf{a})=\mathbf{c}$. Но по выбору скалярного произведения $\langle\mathbf{c}, \mathbf{c}\rangle=\langle\mathbf{a}, \mathbf{a}\rangle=\|\mathbf{a}\|^{2}=\|\mathbf{c}\|^{2}$, поэтому $\langle\mathbf{b}, \mathbf{b}\rangle=\|\mathbf{b}\|^{2}$. Последнее равенство и означает, что норма $\|\cdot\|$ порождена скалярньм произведением $\langle\cdot, \cdot\rangle$. 
ЗАмЕчАниЕ 4.9. Следствие может быть получено также из теоремы Ванга-Титса о классификации связных локально компактных пространств, метрически однородных по отношению к классу всех двухточечных подмножеств. Вопрос о сушественности в этом следствии конечномерности составляет содержание известной задачи Мазура [60; гл. III, §3]. Как указал автору Э.Б. Винберг, теорема 4.6 является также следствием ортогональной представимости компактной подгрупшы общей группы линейных преобразований [61; гл. 12, §2, теорема 3], [62; с. 250], [63; теорема 1]. Здесь уместно указать, что так как пространство всех изометрий метрического компакта само компактно [64], [65; лекция 29, предложение 4], то в следствии 4.8 достаточно требовать, чтобы сохраняюшие нуль изометрии действовали на единичной сфере топологически транзитивно, т.е. имели плотную орбиту.

ЛЕмма 4.10. Если в топологическом векторном пространстве норма (метрика) на каждой двумерной плоскости является евклидовой, то она является евклидовой и на всем пространстве.

ДокАЗАТЕЛЬство. Пусть $a$ и $b$ - два произвольных вектора. Так как в плоскости, порожденной этими двумя векторами, заданная норма порождена некоторым скалярным произведением, то для них справедливо равенство

$$
\|a+b\|^{2}+\|a-b\|^{2}=2\left(\|a\|^{2}+\|b\|^{2}\right) .
$$

Так как векторы $a$ и $b$ произвольны, то по теореме Колмогорова-Шварца [66; гл. $3, \S 4$, теорема 8] заданная норма порождена некоторым скалярным произведением.

СЛЕДСТВИЕ 4.11. Если в нормированном векторном пространстве для всякого равнобедренного треугольника существует изометрия всего пространства, сохраняющая вершину и меняющая местами основания, то оно евклидово.

ДокАЗАТЕльство. Действительно, согласно следствию 4.8 в таком пространстве каждая двумерная плоскость является евклидовой.

ЗАмечАниЕ 4.12. Как мы уже указьвали, теоремы Колмогорова, Буземана и Биркгофа метрически выделяют евклидову, гиперболическую и сферическую геометрии. Евклидова геометрия выделяется из указанных трех геометрий сушествованием подобий. Из универсальности пространств $U$ и $U_{0}$ легко вьвести, что в них всякое подобие компактного подмножества продолжается до подобия всего пространства на себя.

\section{СПИСОК ЛИТЕРАТУРЫ}

[1] Д. Гильберт. Основания геометрии. М.-Л.: Гостехиздат, 1948.

[2] H. Weyl. Mathematische Analyse der Raumproblems. Berlin: Springer-Verlag, 1923.

[3] A. Kolmogoroff. Zur topologisch-gruppentheoretischen Begründung der Geometrie // Nachr. Königl. Gesell. Wiss. Göttingen. 1930. V. 2. P. 208-210.

[4] H. Busemann. On Leibniz's definition of planes // Amer. J. Math. 1941. V. 63. P. 101-111.

[5] H. Busemann. Metric Methods in Finsler Spaces and in the Foundations of Geometry. Princeton: Princeton Univ. Press, 1942. (Ann. of Math. Stud. V. 8.)

[6] G. Birkhoff. Metric foundations of geometry. I // Trans. Amer. Math. Soc. 1944. V. 55. № 3. P. 465-492.

[7] G. Birkhoff. Extensions of Lie groups // Math. Z. 1950. V. 53. № 3. P. 226-235. 
[8] H. Busemann. Recent Synthetic Differential Geometry. Berlin: Springer-Verlag, 1970. (Ergeb. Math. Grenzgeb. V. 54.)

[9] H.-C. Wang. Two theorems on metric spaces // Pacific J. Math. 1951. V. 1. № 3. P. 473-480.

[10] H.-C. Wang. Two-point homogeneous spaces // Ann. of Math. (2). 1952. V. 55. № 1. P. $177-191$.

[11] J. Tits. Étude de certains espaces métriques // Bull. Soc. Math. Belg. 1952 (publié en 1953). V. 5. P. $44-52$

[12] J. Tits. Sur un article précédent: "Étude de certains espaces métriques" // Bull. Soc. Math. Belg. 1953 (publié en 1954). V. 6. P. 126-127.

[13] J. Tits. Sur certaines classes d'espaces homogènes de groupes de Lie. Brussels: Acad. Roy. Belgique, 1955. (Acad. Roy. Belg. Cl. Sci. V. 29.)

[14] H. Freudenthal. Neuere Fassungen des Riemann-Helmholtz-Lieschen Raumproblems // Math. Z. 1956. V. 63. P. 374-405.

[15] H. Freudenthal. Zur Geschichte der Grundlagen der Geometrie // Nieuw Arch. Wisk. (3). 1957. V. 5. P. 105-142.

[16] H. Freudenthal. Die Grundlagen der Geometrie um die Wende des 19. Jahrhunderts // Math.-Phys. Semesterber. 1960. V. 7. P. 1-25.

[17] H. Freudenthal. Zu den Weyl-Cartanschen Raumproblemen // Arch. Math. 1960. V. 11. P. 107-115.

[18] H. Freudenthal. Lie Groups in the foundations of geometry // Adv. Math. 1964. V. 1. № 2. P. $145-190$.

[19] V. H. G. Singh. Two point homogeneous spaces // Proc. Konink. Nederl. Akad. Wetensch. Ser. A. 1965. V. 68. P. 746-753.

[20] H. Freudenthal. Zweifache Homogenität und Symmetrie // Proc. Konink. Nederl. Akad. Wetensch. Ser. A. 1967. V. 70. № 1. P. 18-22.

[21] H. Matsumoto. Quelques remarques sur les espaces riemanniens isotropes // C. R. Acad. Sci. Paris. Sér. A. 1971. V. 272. № 4. P. 316-319.

[22] Дж. Вольф. Пространства постоянной кривизны. М.: Наука, 1982.

[23] А. Л. Онищик. Топология транзитивных групп преобразований. М.: Наука, 1995.

[24] Ф. Бахман. Построение геометрии на основе понятия симметрии. М.: Наука, 1969.

[25] П. С. Урысон. Труды по топологии и другим областям математики. Т. 2. М.: Гостехиздат, 1951.

[26] А. М. Вершик. Универсальное пространство Урысона, метрические тройки Громова и случайные метрики на натуральном ряде // УМН. 1998. Т. 53. № 5. С. 57-64.

[27] С. А. Богатый. Универсальная однородная рациональная ультраметрика на пространстве иррационалшных чисел // Вестник МГУ. Сер. матем., мех. 2000. №6. С. 20-24.

[28] С. А. Богатый. Компактная однородность универсального метрического пространства Урысона // УМН. 2000. Т. 55. №2. С. 131-132.

[29] В.И. Трофимов. Об одном свойстве универсалнного метрического пространства Урысона // Матем. заметки. 2001. Т. 69. № 2. С. 319-320.

[30] Ю. М. Смирнов. Геометрия бесконечных равномерных комплексов и $\delta$-размерность точечных множеств // Матем. сб. 1956. Т. 40. № 2. С. 137-156.

[31] L. M. Blumenthal. Theory and Applications of Distance Geometry. New York: Chelsea, 1970.

[32] L. Janos. A metric characterization of zero-dimensional spaces // Proc. Amer. Math. Soc. 1972. V. 31. № 1. P. 268-270.

[33] С. Д. Илиадис. Компактные и вполне ограниченные метрические пространства и изометрические вложения // УМН. 1995. Т. 50. №6. С. 179-180.

[34] M. Gromov. Groups of polynomial growth and expanding maps // Inst. Hautes Études Sci. Publ. Math. 1981. V. 53. P. 53-78.

[35] Ю. Д. Бураго, В. А. Залгаллер. Введение в риманову геометрию. С.-Петербург: Наука, 1994.

[36] D. Avis. On the extreme rays of the metric cone // Canad. J. Math. 1980. V. 32. №1. P. $126-144$.

[37] S. Watson. The classification of metrics and multivariate statistical analysis // Topology Appl. 1999. V. 99. P. 237-261. 
[38] Е. А. Смоленский. Об одном способе линейной записи графов // Журн. вычисл. матем. и матем. физ. 1962. Т. 2. № 2. С. 371-372.

[39] S. L. Hakimi, S.S. Yau. Distance matrix of a graph and its realizability // Quart. Appl. Math. 1965. V. 22. № 4. P. 305-317.

[40] К. А. Зарецкий. Построение дерева по набору расстояний между висячими вершинами // УМH. 1965. T. 20. №6. C. 90-92.

[41] J. M.S. Simões Pereira. A note on the tree realizability of a distance matrix // J. Combin. Theory. 1969. V. 6. № 3. P. 303-310.

[42] P. Buneman. A note on the metric properties of trees // J. Combin. Theory Ser. B. 1974. V. 17. P. $48-50$.

[43] J. Mayer, J. Nikiel, L. Oversteegen. Universal spaces for $\mathbb{R}$-trees // Trans. Amer. Math. Soc. 1992. V. 334. № 1. P. 411-432.

[44] M. Gromov. Hyperbolic groups // Math. Sci. Res. Inst. Publ. 1987. V. 8. P. 75-263.

[45] M. Gromov. Geometric Group Theory. V. 2: Asymptotic Invariants of Infinite Groups. Proceedings of the symposium held at the Sussex University, Brighton, 1991. Cambridge: Cambridge Univ. Press, 1993. (London Math. Soc. Lecture Note Ser. V. 182.)

[46] M. Culler, P. B. Shalen. Varieties of group representations and splittings of 3-manifolds // Ann. of Math. (2). 1983. V. 117. P. 109-146.

[47] J. W. Morgan, P. B. Shalen. Valuations, trees, and degenerations of hyperbolic structures. I // Ann. of Math. (2). 1984. V. 120. P. 401-476.

[48] M. Bestvina. Degenerations of the hyperbolic space // Duke Math. J. 1988. V. 56. № 1. P. $143-161$

[49] Э. Гис, П. де ля Арп. Гиперболические группы по Михаилу Громову. М.: Мир, 1992.

[50] J. W. Morgan. $\Lambda$-trees and their applications // Bull. Amer. Math. Soc. 1992. V. 26. № 1. P. $87-112$.

[51] B.H. Bowditch. Treelike structures arising from continua and convergence groups // Mem. Amer. Math. Soc. 1999. V. 139. № 662. P. 1-86.

[52] M. Kapovich, B. Leeb. On asymptotic cones and quasi-isometry classes of fundamental groups of 3-manifolds // Geom. Funct. Anal. 1995. V. 5. № 3. P. 582-603.

[53] M. Е. Тылкин (M. Deza). О геометрии Хэмминга единичных кубов // Докл. AH CCCP. 1960. T. 134. № 5. C. 1037-1040.

[54] S. Mazur, S. Ulam. Sur les transformations isométriques d'espaces vectoriels, normés // C. R. Acad. Sci. Paris. 1932. V. 194. P. 946-948.

[55] И. Полтерович, А. Шнирельман. Асимптотический подконус плоскости Лобачевского как пространство функций // УМН. 1997. Т. 52. № 4. С. 209-210.

[56] А.Г. Дюбина, И.В. Полтерович. Структуры на бесконечности гиперболических пространств // УМН. 1998. Т. 53. № 5. С. 239-240.

[57] D. van Dantzig. Über topologisch homogene Kontinua // Fund. Math. 1930. V. 15. P. $102-125$.

[58] F. John. Extremum problems with inequalities as subsidiary conditions // Studies and essays, presented to R. Courant on his 60th birthday. New York, 1948. P. 187-204.

[59] К. Лейхтвейс. Выпуклые множества. М.: Наука, 1985.

[60] С. Улам. Нерешенные математические задачи. М.: Наука, 1964.

[61] Э. Б. Винберг. Курс алгебры. М.: Факториал, 1999.

[62] B. Huppert. Angewandte lineare Algebra. Berlin: de Gruyter, 1990.

[63] H. Rummler. On two characteristic properties of Euclidean norms // Elem. Math. 1991. V. 46. P. 111-115.

[64] D. van Dantzig, B. L. van der Waerden. Über metrisch homogene Räume // Abh. Math. Hamburg. Univ. 1928. V. 6. P. 367-376.

[65] М. М. Постников. Риманова геометрия. М.: Факториал, 1998.

[66] А. Н. Колмогоров, С. В. Фомин. Элементы теории функций и функционального анализа. М.: Наука, 1976. 